\title{
Vorwort zur 109. Auflage
}

Die Automatisierung und Digitalisierung haben in den letzten Jahren sowohl in der klassisch chemischen Analytik, der instrumentellen Analytik als auch bei vielen physikalisch chemischen Messverfahren immense Fortschritte erzielt. Es besteht die Gefahr, dass Analysengeräte nur noch als „Black Box“ wahrgenommen werden, die die gewünschten Daten liefern. Der „Küster-Thiel“ hat sich auch in der vorliegenden Auflage wieder zum Ziele gesetzt, in der Ausbildung zum grundlegenden Verständnis von Analysenverfahren, Methodik und Auswertung beizutragen.

Am 20. Mai 2019 ist eine historische Änderung weltweit in Kraft getreten, die die SI-Basiseinheiten auf eine neue Grundlage stellt. Erstmals ist es gelungen alle 7 Basiseinheiten auf 7 Naturkonstanten zu beziehen. Die Definitionen der SI-Basiseinheiten wurden entsprechend angepasst. Desweiteren wurden die Kapitel Temperaturmessung und Kalibrierung von Volumenmessgefäßen überarbeitet. Neu eingefügt wurden Daten und Rechenbeispiele für grundlegende thermodynamische Größen wie Energie, freie Energie und Entropie.

Für die ausgezeichnete Zusammenarbeit mit Frau Dr. Fritz und Frau Karola Seitz vom Verlag de Gruyter möchten wir uns herzlich bedanken.

Schriesheim im Juli 2019

Ursula Ruland

Alfred Ruland 